Voix et Images

voixetimages

\title{
Le discours intellectuel
}

\section{Robert Major}

Volume 19, numéro 2 (56), hiver 1994

\section{Anne-Marie Alonzo}

URI : https://id.erudit.org/iderudit/201099ar

DOI : https://doi.org/10.7202/201099ar

Aller au sommaire du numéro

\section{Éditeur(s)}

Université du Québec à Montréal

\section{ISSN}

0318-9201 (imprimé)

1705-933X (numérique)

Découvrir la revue

\section{Citer cet article}

Major, R. (1994). Le discours intellectuel. Voix et Images, 19(2), 396-403.

https://doi.org/10.7202/201099ar d'utilisation que vous pouvez consulter en ligne.

https://apropos.erudit.org/fr/usagers/politique-dutilisation/ 


\section{Essai}

\section{Le discours intellectuel}

\section{Robert Major, Université d'Ottawa}

Ce titre est pléonastique, évidemment. L'intellectuel n'est que discours. Le médecin peut parler de la maladie; l'ingénieur, des ponts et chaussées; le jardinier, de son potager; le prêtre, de Dieu. Chacun parle d'un objet, plus ou moins défini, aux contours précis ou flous, selon le cas, mais néanmoins extérieur à soi, dissociable, autre. Et quand ces gens ne discourent pas, ils agissent, chacun dans sa sphère propre, raccommodant des corps, rasant des forêts, récoltant des choux, sauvant des âmes. L'intellectuel, lui, quand il veut agir, fonde une revue! Pour mieux discourir.

Cela n'est pas nécessairement infamant ou futile. Ne sait-on pas depuis saint Jean que le Verbe est à l'origine de toute chose: "Tout fut par lui et sans lui rien ne fut" (Jean I, 3)? Reconnaître le pléonasme relève simplement de l'ordre de la constatation: l'intellectuel est discours. Discours sur la réalité, certes, mais surtout sur la perception de celle-ci par les autres intellectuels. Ainsi, seul l'intellectuel s'intéresse, dans sa pratique savante, à lui-même; c'est-àdire, à "d'autres lui-mêmes". Dans le meilleur des cas, à d'autres manieurs d'idées, fabricants de théories, faiseurs de systèmes, énonciateurs de paradigmes, bricoleurs de programmes, inventeurs de slogans; dans la plupart des cas, malheureusement, à d'autres répétiteurs épigoniques d'idées reçues ou de lieux communs, les mots suscitant des mots, les idées, d'autres idées, le plus souvent empruntés. Seul l'intellectuel s'intéresse à la vie intellectuelle, réalité imprécise s'il en est, et aux autres intellectuels. Et, s'y intéressant, il se trouve forcément à s'insérer de plain-pied dans la "boulimie discursive" du temps, comme dirait François Ricard, et à contribuer à la "conflagration spéculative" (toujours Ricard), propre à ce commerce, surtout à notre époque où les mots ne coûtent rien.

Peut-on sortir de ce cercle vicieux, sinon épistémologique, du moins profondément narcissique? D'ailleurs, veut-on sortir du cercle? Il faudrait que dès cet instant je pose la plume et que j'invite mes 
collègues à saborder notre propre revue! Le silence, enfin. Mais le désert, aussi. Le veut-on?

Et est-ce qu'on sort du cycle infernal du seul fait de son titre de sociologue, le titre étant supposé garant d'une quelconque scientificité qui permettrait d'étudier avec détachement et rigueur les "productions signifiantes" de ses alter ego?

Réflexions qui me viennent en prenant en main le livre d'Andrée Fortin: Passage de la modernité. Les intellectuels québécois et leurs. revues $^{1}$, et d'autant plus que l'auteure affirme d'emblée, à la fin de son introduction, que son intérêt pour les revues et les intellectuels qui les animent vient de ses liens étroits avec différents animateurs de revues et de sa participation effective aux comités de rédaction de différentes publications. La distance critique s'amenuise dangereusement. Mais peut-être, aussi, est-ce ainsi qu'on pratique la sociologie aujourd'hui: en contribuant aux activités qu'on s'apprête à analyser; je n'en sais rien.

Quoi qu'il en soit, l'entreprise est intéressante. Le projet, en effet, est à la fois ambitieux et précis: prendre l'éditorial initial, le manifeste liminaire, la présentation ou le prospectus de cinq cent seize revues québécoises qui ont existé entre 1778 et 1989 (celles que l'auteure a pu consulter: environ la moitié des revues du $\mathrm{XIX}^{\mathrm{e}}$ siècle et presque la totalité de celles du $\mathrm{xx}^{\mathrm{e}}$ ); étudier ce "premier éditorial " comme un genre précis, aux contours définis, aux visées analogues; en dégager les indices de l'insertion des intellectuels dans la cité, c'est-à-dire les modalités (ou du moins les modalités postulées!) de l'activité discursive des intellectuels dans leur société. En effet, le moment de la fondation d'une revue, la première parole d'un nouveau "Nous" qui s'affirme sur la place publique et du même coup affirme un "vide qu'il s'agit de combler" (p. 10) - puisque les autres revues n'occupent pas cet espace -, qui se définit donc en se situant face à la réalité ambiante, face aussi à son public ou à ses adversaires, semble à l'auteure, et avec raison, un moment privilégié de la vie intellectuelle d'une collectivité. Reconnaissons d'emblée que ce matériel est riche et significatif.

Si le projet est intéressant, sa réalisation, toutefois, ne nous satisfait pas complètement. On serait en peine de dire exactement pourquoi. Est-ce parce que l'auteure embrasse trop large et que prétendre dresser le portrait d'une certaine vie intellectuelle à partir d'un nombre si considérable de revues confinait à l'impossible, à moins d'effectuer une sélection clairement motivée? Est-ce parce que l'objet est. mal cerné et qu'il aurait fallu mieux cibler, ce qui aurait permis de déterminer exactement ce qu'est une revue intellectuelle? Est-ce parce que 
cette étude trouve mal son créneau, écartelé entre l'enquête ou l'inventaire sociologique, qui aurait exigé une approche beaucoup plus rigoureuse, et l'essai, qui vraisemblablement autorise l'auteure à y aller plutôt librement dans ses ponctions? Car l'étude, dans sa forme actuelle, n'est ni l'un ni l'autre, et risque de ne satisfaire personne. Estce l'imprécision méthodologique, qui a conduit l'auteure à alterner entre un parcours chronologique et des regroupements thématiques, ces derniers étant tout à fait inadéquats pour saisir le XIx ${ }^{e}$ siècle, et d'autant plus que l'auteure s'intéresse aux transformations (donc aux modifications diachroniques) du rapport des intellectuels avec le socio-politique? Sans doute tous ces aspects sont-ils en cause.

Il faut préciser, toutefois, que les revues en elles-mêmes n'intéressent pas la sociologue. Elles ne sont qu'un "prétexte" (passim). Son but, clairement affirmé dès la première phrase, est de cerner le rapport des intellectuels québécois avec le social et le politique et même, de façon plus étroite, de comprendre l'état actuel de ce rapport. L'étude, en effet, a en quelque sorte un but polémique (d'où mes reserves: sur la distance critique): démontrer que les intellectuels contemporains ne sont pas silencieux. Ils sont pleinement insérés dans le social d'aujourd'hui. Leur perception de. la société, toutefois, et le type de rapport qu'ils entretiennent avec le politique a changé radicalement.

Pour justifier cette conviction, un long parcours historique semble s'imposer à l'auteure, parcours conduit par l'hypothèse suivante: il y a eu différenciation progressive, à partir du $\mathrm{xIx}^{\mathrm{e}}$ siècle, des sphères politique et intellectuelle. $\mathrm{Au} \mathrm{XIX}^{\mathrm{e}}$ siècle, l'intellectuel est dans la sphère politique et ne s'en distingue guère. Dans la première partie du $\mathrm{xx}^{\mathrm{e}}$ siècle, une différenciation s'effectue: l'intellectuel s'affirme comme le précurseur et croit que la politique est à sa remorque, ou du moins à la remorque des idées. Dans la période actuelle (l'auteure situe la brisure en 1979), les deux sphères sont résolument distinctes. On laisse la politique aux politiciens. Le politique, toutefois, prend de nouvelles significations, postmodernes. Il préoccupe toujours autant les intellectuels, toutefois, qui ne sont ni silencieux, ni démissionnaires.

Il y a donc, dans cette étude, une espèce d'apologie des intellectuels de notre temps. Ils sont toujours là, actifs et remuants. Ils n'ont pas choisi le silence. Ils ont simplement d'autres paradigmes. En effet, la modernité est passée par là (et sans doute est-ce le sens du titre, "passage de la modernité"), changeant l'horizon et les conditions de la vie intellectuelle. Le féminisme, l'interculturel, la marginalité de tout ordre (géographique, idéologique, sexuelle, littéraire, artistique...) font 
éclater la collectivité ou, peut-être, cherchent à la redéfinir par le biais de la vie privée ou du transculturel. Les intellectuels ne s'occupent plus des enjeux globaux, ni de la totalité québécoise. Ils sont devenus spécifiques, braqués sur des problèmes concrets, quotidiens, privés. Il y a donc eu mutation. L'intellectuel ne parle plus au nom de la collectivité, ne cherche plus à cerner la réalité du Nous québécois, ses conditions d'existence et ses possibilités d'épanouissement. Il fonde maintenant une revue pour se faire plaisir, ou pour promouvoir les intérêts ponctuels, limités et partiels d'un petit collectif.

Sommes-nous toujours dans la sphère du social et du politique? Sans doute, et sans doute une sociologue a-t-elle raison de s'intéresser à cette mutation, qui est un phénomène social. Mais on pourrait légitimement se poser la question: tous ces petits Nous privés qui pullulent présentement, qui fondent des revues aux ambitions restreintes, qui prennent donc la parole pour se faire l'écho des mouvements sociaux en cours plutôt que pour les créer ou les analyser, sontils bien des intellectuels? Fonder une revue est certainement "l'acte intellectuel par excellence" (p. 9), mais suffit-il de fonder une revue pour faire acte intellectuel? Fonder une revue n'exige aujourd'hui, on le sait, qu'un ordinateur et quelques loisirs.

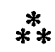

Les fondateurs et animateurs de revues - et leurs commentateurs? - peuvent facilement avoir l'impression qu'ils sont à la source et à la fine pointe de l'activité intellectuelle et que leurs propos sont essentiels à la collectivité. Ils oublient facilement qu'un travail d'un autre type, moins fracassant, moins visible mais peut-être plus déterminant s'effectue dans d'autres milieux, par exemple dans les universités. Jean Lamarre nous en sert une démonstration magistrale dans son livre récent: Le Devenir de la nation québécoise ${ }^{2}$. Dans son étude aussi il est question de modernité et de redéfinitions radicales de la collectivité québécoise, mais. Jean Lamarre s'oppose au "mythe des origines" de la modernité québécoise qui ferait commencer celle-ci avec, par exemple, le combat "héroïque " de Cité libre contre les forces de l'obscurantisme et en faveur de la modernité et de la démocratie sociale. Sans minimiser le combat des ténors des revues ou des journaux (par exemple Le Devoir), il met en lumière le travail des universitaires, travail obscur en salle de classe ou dans de savantes monographies, activités, donc, le plus souvent éloignées de la place publique mais dont l'effet est profond et prolongé. 
Son livre est touffu, et même d'une redoutable densité, mais il se lit comme un roman. Cela tient à ses propres qualités de présentation claire et méthodique d'une matière abondante et complexe; mais cela tient, également, à l'intérêt intrinsèque de son sujet et à la qualité des protagonistes qui sont mis en scène. On y voit que l'histoire, aussi, est un discours et les historiens de redoutables discoureurs. Ceux qui n'en seraient pas convaincus et qui croiraient, par exemple, que l'histoire est une science, seront édifiés par sa lecture. Ce livre, en effet, nous présente un excellent bilan de tous les discours tenus sur le Canada français par les historiens et nous offre in compte rendu captivant des polémiques furieuses que se sont livrés ceux-ci pour faire admettre leur vision particulière.

L'objet propre de l'étude est de présenter les historiens de l'École de Montréal, c'est-à-dire Maurice Séguin, Guy Frégault et Michel Brunet, qui ont commencé à enseigner à l'Université de Montréal à la fin des années quarante et dont l'œuvre historiographique a profondément bouleversé la perception que le Canada français pouvait se faire de son histoire et, partant, de son identité. Une première partie en deux chapitres trace les grands traits de la tradition historiographique au tournant des années quarante, tant du côté canadien-anglais que canadien-français. On y trouve un excellent résumé de chacune de ces traditions et une mise en relief de la contribution des historiens marquants. Chacune des parties suivantes est consacrée à l'un des historiens de l'École de Montréal, faisant ressortir son itinéraire personnel et intellectuel avant d'effectuer une analyse des œuvres et de leur retentissement. Les ouvres sont analysées en elles-mêmes, les unes par rapport aux autres et par rapport, également, à la tradition qu'elles intègrent ou qu'elles font éclater.

Ces trois historiens, malgré des différences notables de personnalité et des parcours personnels fort différents, présentent une réflexion d'une remarquable cohérence, justifiant qu'on puisse parler d'école et de doctrine. Cette réflexion s'articule sur l'événement de la Conquête et le poids de l'histoire dans le devenir d'une nation. La Conquête est une défaite militaire, certes, mais davantage: la société canadiennefrançaise a été littéralement dé-faite, c'est-à-dire disloquée, brisée, démembrée, désorganisée par cet événement. Tout le reste en découle.

L'infériorité économique dẹs Canadiens français, le caractère rétrograde de leurs institutions, l'influence disproportionnée qu'exerce le clergé au sein de la société, ainsi que l'idéologie de la survivance nationale ne sont que les conséquences diverses de la rupture qu'a provoquée la Conquête anglaise dans le devenir de la nation canadienne-française (p. 19).

Avec un tel point de vue sur l'histoire, on comprend aisément que ces universitaires se soient mis à dos tout le monde: le clergé, les 
propagandistes d'une histoire légendaire, providentielle et messianique, les partisans d'une bonne entente avec les Canadiens anglais, les citélibristes qui croyaient que les Canadiens français étaient euxmêmes responsables de leurs retards, les éternels optimistes qui croyaient tout possible et même les amateurs d'histoire qui y cherchent, essentiellement, un réservoir de figures admirables.

Cette doctrine ne s'est pas constituée subitement et elle n'a pas été articulée de façon équivalente par chacun des historiens. C'est le grand mérite du livre de Jean Lamarre que d'être extrêmement attentif aux personnes, aux événements et à la singularité des ouvres. C'est ainsi qu'il nous montre la genèse de cette vision, d'abord dans la réflexion laborieuse de Maurice Séguin, dont le rôle séminal est déterminant; puis son déploiement, dans la production abondante de Guy Frégault, dont l'œuvre complexe est patiemment analysée; finalement, sa diffusion, dans les combats et les interventions publiques de Michel Brunet, toujours prêt à monter aux barricades et convaincu, d'ailleurs, que tout n'était pas joué et que la collectivité pouvait encore tirer son épingle du jeu, si elle voulait bien exploiter les instruments à sa disposition, en particulier l'État du Québec.

Cette attention aux ouvres et aux destins singuliers permet de recevoir avec équanimité la boutade qu'aurait commise Mason Wade à l'effet que l'École de Montréal serait formée "d'un qui pense, d'un qui écrit, et d'un qui crie" (p. 384). Comme toute caricature, celle-ci recèle sa part de vérité. Il y a un contraste très net entre Maurice Séguin, qui n'a quasi rien publié; Guy Frégault, dont la production intense, précoce et de qualité force l'admiration, et Michel Brunet, homme d'action, de combat et de parole. Par ailleurs, chaque ouvre, peu importe ses dimensions, est abordée par Jean Lamarre avec le plus grand respect, et saisie dans son originalité propre. Monographie, conférence, notes de cours, correspondance, tous les éléments sont mis à contribution pour réaliser le tableau vivant de cette période particulièrement féconde, celle des décennies quarante et cinquante, qui précèdent, préparent et disposent la Révolution tranquille.

Et ce n'est pas le moindre intérêt de ce tableau que d'y voir, bien dessiné à l'arrière-plan, la grande figure de Lionel Groulx. Il est un personnage omniprésent dans ce livre, non seulement de par l'influence de son ouvre qui en fait la référence obligatoire, mais aussi de par son influence personnelle. Il est le maitre et le parrain, celui qui prépare, favorise et fabrique des carrières, celui qui choisit et lance ses successeurs puis qui assiste à leurs combats, mi-amusé, mieffarouché, mais toujours encourageant, honnête et résolument 
respectueux de l'itinéraire intellectuel de ses protégés, devenus ses collègues. Ce respect ira jusqu'à la reconnaissance de l'obligation de réviser ses propres jugements historiques (voir.p. 409). Le maitre se met à l'école de ses élèves, suprême consécration, et indication de l'effet considérable qu'aura la vision historique de cette École dans les années à venir. Et c'est ainsi que le titre de cette étude prend toutes ses résonances. Le "devenir " de la nation québécoise, c'est celui qui était possible, systémiquement, étant donné l'événement traumatisant de la Conquête: c'est celui que le Canada français, société dé-faite, vivait depuis lors. Mais ce "devenir" est aussi celui qui sera devenu possible quand la collectivité aura accepté cette vision des choses, aura reconinu ce qu'elle est réellement, et cherchera à se re-faire.

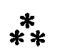

Le discours de l'historien grave et austère a-t-il plus d'influence sur la vie intellectuelle d'une collectivité que celui d'un animateur de revue dite intellectuelle? Difficile de répondre à la question, et d'autant plus que nos historiens, qu'ils soient de l'École de Montréal ou qu'ils s'appellent Lionel Groulx, Marcel Trudel ou Fernand Ouellet, n'ont rien des universitaires désincarnés ou sereins. Par leurs travaux, ils cherchent à convaincre. L'histoire est toujours rhétorique et expression d'une vision du monde. Et si la monographie ne suffit pas, la conférence ou le compte rendu servira tout aussi bien à démolir l'adversaire.

D'ailleurs, peut-on aisément distinguer l'universitaire de l'animateur de revues? Guy Frégault, jadis, se moquait, d'une plume vitriolique, des revues de son temps:

Voyez notre "élite" intellectuelle. Elle est amusante. Voyez nos petites revues dites d'avant-garde. Elles sont sympathiques. Mais ne vous semble-t-il pas que ces beaux esprits sont tous nés vulgarisateurs des vulgarisateurs, commentateurs des commentateurs, échos des échos? (p. 258-259)

Ces propos datent de 1944. Je ne les cite pas, toutefois, pour illustrer leur remarquable pertinence actuelle. Plutôt pour signaler que Guy Frégault, dès l'année suivante, et sans délaisser sa chaire universitaire, prenait la direction de L'Action nationale! Toutes les tribunes sont à saisir quand les intellectuels veulent discourir.

1. Andrée Fortin, Passage de la modernité. Les intellectuels québécois et leurs revues, Sainte-Foy, Les Presses de l'Université Laval, 1993, 406 p. 
2. Jean Lamarre, Le Devenir de la nation québécoise selon Maurice Séguin, Guy Frégault et Michel Brunet 1944-1969, Sillery, Les Éditions du Septentrion, 1993, $564 \mathrm{p}$. 